\title{
Antifungal Properties of Silver Nanoparticles Synthe- sized From Capparis Spinosa Fruit
}

\author{
Katrin Ebrahimi $^{*}$ (D, Mahboobeh Madani ${ }^{2}$ (D), Behnam Ashrafi ${ }^{3}$ (D, Sima Shiravand ${ }^{4}$ (D, Asghar Sepahvand SiD $^{3}$
}

1. Department of Biology, Faculty of Science, Payam Noor University, Tehran, Iran.

2. Department of Microbiology, Islamic Azad University, Falavarjan Branch, Isfahan, Iran.

3. Razi Herbal Medicines Research Center, Lorestan University of Medical Sciences, Khorramabad, Iran.

4. Department of Biology, Faculty of Science, Lorestan University, Khorramabad, Iran.

\begin{tabular}{|l|l|}
\hline $\begin{array}{l}\text { Use your device to scan } \\
\text { and read the article online }\end{array}$ & $\begin{array}{l}\text { testion Ebrahimi K, Madani M, Ashrafi B, Shiravand S, Sepahvand A. Antifungal Properties of Silver Nanoparticles Syn- } \\
\text { thesized From Capparis Spinosa Fruit. Research in Molecular Medicine. 2019; 7(4):43-50. https://doi.org/10.32598/rmm.7.4.43 }\end{array}$ \\
\hline
\end{tabular}

\section{c) (i)}

Article Type:

Research Article

Article info:

Received: 27 Aug 2019

Revised: 1 Oct 2019

Accepted: 18 Oct 2019

Keywords:

Antifungal activity,

Aqueous extract,

Characterization,

Capparis spinosa, Silver

nanoparticle

\begin{abstract}
A B S T RA C T
Background: Nanoparticles (NPs) are colloidal systems with particles ranging from 10 to $100 \mathrm{~nm}$ in diameter. Because of their large surface-volume ratio, NPs are biologically active materials that could interact with biomolecules and microorganisms, enter into the cells, and affect the metabolic functions. The study aimed to biosynthesize silver nanoparticles (Ag-NPs) from Capparis spinosa fruit aqueous extract, and evaluate their Ag nanostructure characterization and antifungal activity.

Materials and Methods: Capparis spinosa fruit aqueous was prepared with the percolation method. Then, silver NPs were synthesized using $0.01 \mathrm{M}$ silver nitrate solution, and their formation was validated by color changing of the solution from green to dark brown. The NPs were purified using centrifugation and then dried in an oven for further analyses. Ag-NPs nanostructure characterization was determined by various techniques such as Fourier Transforms Infrared (FTIR) spectroscopy, Scanning Electron Microscope (SEM), and Ultraviolet-visible (UV-Vis) spectroscopy. Antifungal activity of Ag-NPs against three pathogenic fungi of Candida albicans, Candida glabrata, and Kluyveromyces marxianus was also evaluated using the microdilution method.
\end{abstract}

Results: Synthesis of Ag-NPs from aqueous extract of C. spinosa fruit was done successfully. UVVis spectrum of Ag-NPs showed an absorbance peak around $420 \mathrm{~nm}$, revealing Ag-NPs surface plasmon resonance $\left(\mathrm{K}_{\max }\right)$. FTIR analysis showed that functional groups correspond to plant bioactive components, promoting the formation of Ag-NPs. Furthermore, spherical uniformity of the synthesized Ag-NPs from plant extract was confirmed by SEM analysis within the $50-80 \mathrm{~nm}$ size range. Our results showed that the produced Ag-NPs were spherical and in a suitable form and size $(50-80 \mathrm{~nm})$. The biosynthesized Ag-NPs had an inhibitory effect against all tested fungi with the minimum inhibitory concentration of 2500,5000 , and $625 \mu \mathrm{g} / \mathrm{mL}$ and minimum bactericidal concentration of 10000,10000 , and $156.25 \mu \mathrm{g} / \mathrm{mL}$ for C. albicans, C. glabrata, and K. marxianus, respectively.

Conclusion: According to the UV-Vis spectrum, FTIR, and SEM results, we succeeded in synthesizing Ag-NPs from C. spinosa fruit aqueous extract. This research was the first report of Ag-NPs synthesized from aqueous extract of $C$. spinosa fruit. Our simple, quick, and inexpensive method for biosynthesis of a nanoparticle, which showed antifungal activity, provides a new potential antifungal agent for therapeutic applications.

\footnotetext{
* Corresponding Author:

Katrin Ebrahimi, PhD.

Address: Department of Biology, Faculty of Science, Payam Noor University, Tehran, Iran.

Phone: +98 (916) 1604010

E-mail:ebr_k@yahoo.com
} 


\section{Introduction}

andida albicans is an opportunistic organ-

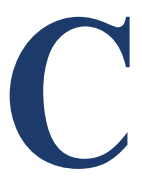
ism which can cause a variety of infections in human. Three major forms of candidiasis are vulvovaginal candidiasis, invasive candidiasis, and oropharyngeal candidiasis. More than $75 \%$ of women suffer from $C$. albicans infection once in their lifetime. It is the fourth leading cause of nosocomial infections in patients' bloodstreams [1]. Although Kluyveromyces marxianus is not usually a pathogen for human disease, it has been recovered from catheters, associated with candit demia, and found in biofilms on many indwelling dee vices such as prosthetic heart valves and pacemakers. $K$. marxianus has been seen in about $1 \%$ to $3 \%$ of cases with female genital infections, surgical wounds, and infections related to upper respiratory tracts $[2,3]$.

Candida glabrata has been introduced as the second most common cause of candidiasis, with the mortality of $40 \%-50 \%$. Infections caused by C. glabrata have increased worldwide, which may be due to the prevalence of antifungal drug resistance among the clinical isolates of this yeast [4]. It is, thus, crucial to assay and apply new drugs to design more suitable therapeutic strategies and control diseases caused by the fungi. Nanoparticles (NPs) are colloidal systems with particles ranging from 10 to $100 \mathrm{~nm}$. Nowadays, a wide range of NPs are available and often produced by chemical and biological methods [5]. Studies have shown that NPs are biologically active due to their large surface-volume ratio. Therefore, they could interact with biomolecules and microorganisms, enter into the cells, and affect their metabolic functions [6].

Recently, silver has drawn considerable attention due to its application in various industries such as jewelry, medicine, electronics, and chemical synthesis as a catalyst. Additionally, the pharmacological properties of the silver NPs persuaded researchers to study Ag-NPs applications in drug delivery, drug design, medical imaging, and controlling antibiotic-resistant pathogens [7]. AgNPs are now synthesized by various methods, including the chemical reduction of Ag ions in solutions, photoreduction in reverse micelles, electrolysis technique, and radiation-assisted reduction $[8,9]$. However, the majority of these methods are not eco-friendly; hence, their use is limited in medicine [10]. These limitations call for the development of new methods for the synthesis of AgNPs with low toxicity and high efficacy.
Green synthesis refers to the use of biological molecules for fabricating various nanostructures. In these methods, biomolecules play a central role in reducing, capping, and stabilizing the produced nanostructures. Since biomolecules are known as biocompatible and degradable molecules, green synthesis of nanomaterials is currently growing [11]. Herbal compounds can potentially reduce metal ions and produce NPs ranging from nanoscale to microscale in a one-step process. Besides, plant-derived NPs are known as non-toxic and eco-friendly compared with those made through physical and chemical methods $[11,12]$.

C. spinosa, with the common name "caper" (Capparidaceae family), grows in the different areas and especially in Iran [13]. Studies have shown that caper contains some potent polyphenols such as quercetin, kaempferol, and anthocyanins with therapeutic effects. Therefore, the caper is extensively used in traditional medicine to treat rheumatism, gastrointestinal disorders, headache, toothache, as well as kidney and liver diseases $[13,14]$ In recent studies, several biological functions, including antioxidant, anticancer, antimicrobial, and anti-inflammatory activity, are related to C. spinosa plant [14]. The present study was conducted to fabricate silver NPs mediated by $C$. spinosa fruit extract and investigate the antifungal activity of produced NPs.

\section{Materials and Methods}

\section{Collection of the plant}

C. spinosa fruits were collected from Kouhdasht City, Lorestan Province, Iran, in 2019 September. They were identified by expert botanists of Razi Herbal Medicine Research Center, Lorestan University of Medical Sciences (Khorramabad, Iran). The fruits were washed using distilled water, shadow dried, powdered, and stored in the refrigerator for later uses.

\section{Preparation of aqueous extract}

Five grams of C. spinosa fruit powder was poured into 75 $\mathrm{mL}$ of deionized water in a $250-\mathrm{mL}$ flask. Then, the flask was stored at $80^{\circ} \mathrm{C}$ for $50 \mathrm{~min}$, and after cooling, the content was filtrated through Whatman filter paper (pore size: 20-25 mm). The filtrated extract with a final concentration of $2 \mathrm{~g} / \mathrm{L}$ stored at $4^{\circ} \mathrm{C}$ until further use $[15,16]$.

\section{Synthesis of silver NPs}

First of all, $60 \mathrm{~mL}$ of freshly prepared extract was added to $100 \mathrm{~mL}$ of freshly prepared $0.01 \mathrm{M}$ silver nitrate 
solution while being stirred continuously, and then it was kept at $60^{\circ} \mathrm{C}$ for $24 \mathrm{~h}$. Color changing of the solution from green to dark brown indicated the formation of NPs. Afterward, it was centrifuged at $10000 \mathrm{rpm}$ for $10 \mathrm{~min}$. Centrifugation procedure was repeated twice to remove all impurities from the synthesized NPs. The resulting purified silver NPs were dried in an oven at $40^{\circ} \mathrm{C}$ and then kept for further analyses [17, 18].

\section{Characterization of silver NPs}

To characterize silver NPs, we used three analytical procedures:

1. Ultraviolet-Visible (UV-Vis) spectrum analysis: $\mathrm{Ag}^{+}$ions reduction was monitored using $\mathrm{UV}-\mathrm{Vis}$ spectrum measurement of the reaction medium after 5 hours. A small aliquot of the sample was diluted in distilled water, and UV-visible spectral analysis (200 to $700 \mathrm{~nm}$ ) was done using a UV-Vis spectrophotometer (JENWAY6405).

2. Fourier Transforms Infrared Spectroscopy (FTIR): The synthesized Ag-NPs and KBr powder were mixed and then pressed into a pellet for measurement. Background correction was prepared using a reference blank $\mathrm{KBr}$ pellet. The analysis and measurement were performed using the FTIR spectrophotometer (model Nicolet 32 ) in a range of 400-4000 and with a resolution of 1-4 $\left(\mathrm{cm}^{-1}\right)$ [18].

3. Scanning Electron Microscope (SEM): Size and morphology of synthesized NPs were examined using electron microscopy (Mira3, Made in Czech) with 15 $\mathrm{kV}$, magnification of $\mathrm{x} 10$, and resolution of $1 \mathrm{~nm}$.

\section{Antifungal activity study}

\section{Fungal strains}

Standard strains of Candida glabrata (PTCC 5297), Candida albicans (PTCC 5072), and Kluyveromyces marxianus (PTCC 5193) were purchased from Departe ment of Medical Mycology, Iran University of Medical Sciences, Tehran, Iran. The strains were incubated in sabouraud dextrose agar at $30^{\circ} \mathrm{C}$ for 2-3 days.

\section{In vitro antifungal activity assessment:}

Antifungal effects of Ag-NPs against the three fungi were investigated using the broth microdilution method, according to the modified M27-A3 protocol on yeasts provided by the Clinical and Laboratory Standards Institute (CLSI, 2005). First of all, two-fold se- rial dilutions of each nanoparticle (ranging from 9.7 to $5000 \mu \mathrm{g} / \mathrm{mL}$ ) were prepared in $1 \%$ dimethyl sulfoxide. After that, $100 \mu \mathrm{L}$ of nanoparticle solution was pipetted into the first row of the 96-well microplates. Then, 50 $\mu \mathrm{L}$ peptone dextrose broth (Sigma-Aldrich) was added to the other wells.

Serial dilutions were prepared similarly for each well that had $50 \mu \mathrm{L}$ of the test material in serially descending concentrations. Afterward, $50 \mu \mathrm{L}$ of broth, containing each one of fungi suspension $\left(5.0 \times 10^{5} \mathrm{CFU} / \mathrm{mL}\right)$, was added to each well. The microplates were sealed with clean film and incubated at $30^{\circ} \mathrm{C}$ for $36 \mathrm{~h}$. Finally, $15 \mu \mathrm{L}$ of 2, 3, 5-triphenyl tetrazolium chloride (TTC, $0.18 \%$ ) solution was added to each well and incubated at $37^{\circ}$ $\mathrm{C}$ for $1 \mathrm{~h}$. The lowest nanoparticle concentration that showed no color change (clear) was recorded as positive and considered as minimum inhibitory concentration (MIC). The lowest concentration that yielded negative sub-cultures was considered as Minimum Bactericidal Concentration (MBC) [19, 20].

\section{Statistical analysis}

All experiments were conducted in triplicate, and the values were analyzed in SPSS V. 19 to find any significant differences among the parameters and variables. Results were considered significant if $\mathrm{P}<0.05$.

\section{Results}

UV-Vis absorption spectroscopy analysis:

The addition of aqueous extract of $C$. spinosa into the beakers containing an aqueous solution of $\mathrm{AgNO}_{3}$ led to color changing of the solution to yellowish to reddishbrown, because of excitation of surface plasmon vibrations in silver NPs. The formation of the color was for excitation of the surface plasmon vibrations, typical of Ag-NPs with גmax values, which are recorded in the visible range of 400-450 nm. Bioactive compounds, like polyphenols, are strong stabilizers and anti-aggregating agents.

Therefore, binding of Ag-NPs to polyphenols creates a significant absorption at $420 \mathrm{~nm}$, indicating how the nanostructure of $\mathrm{Ag}$ is stabilized at the contact of plantderived compounds [21]. Our synthesized NPs also had a maximum peak in the zone of $420 \mathrm{~nm}$ (Figure 1).

\section{Fourier transform infrared analysis:}

C. spinosa fruit extract showed several characteristic bands in the FTIR (Figure 2.B). The peak at 3381.33 
Table 1. Antifungal effects of Ag-NPs (with particle size from 50 to $80 \mathrm{~nm}$ and concentration of 9.7 to $5000 \mu \mathrm{g} / \mathrm{mL}$ ) and fluconazole against some pathogenic fungal strains

\begin{tabular}{ccccc}
\hline & \multicolumn{3}{c}{ Ag-NPs } & \multicolumn{2}{c}{ Fluconazole } \\
\cline { 2 - 5 } Microorganism & MIC $(\mu \mathrm{g} / \mathrm{mL})$ & MBC $(\mu \mathrm{g} / \mathrm{mL})$ & MIC $(\mu \mathrm{g} / \mathrm{mL})$ & $\mathrm{MBC}(\mu \mathrm{g} / \mathrm{mL})$ \\
\hline C. albicans (PTCC 5072) & 2500 & 10000 & 64 & $<128$ \\
C. glabrata (PTCC 5297) & 5000 & 10000 & 128 & 128 \\
K. marxianus (PTCC 5193) & 625 & 156.25 & 128 & $>128$ \\
\hline
\end{tabular}

Abbreviations: MIC, minimum inhibitory concentration; MBC, minimum bactericidal concentration.

काPm

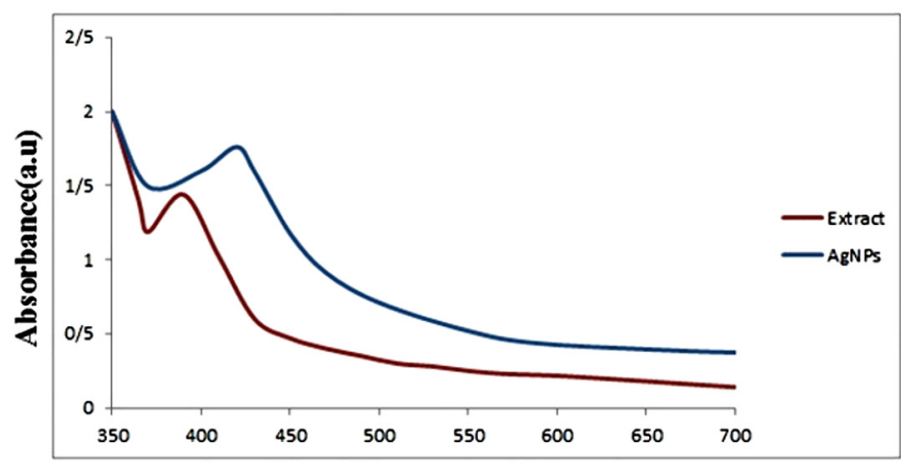

Wavelenght(nm)

Brmm

Figure 1. Ultraviolet-visible spectra of Ag-NPs synthesized (blue) and crude extract of C. spinosa with a concentration of $5 \mathrm{~g} / 75$ $\mathrm{mL}$ (red)

$\mathrm{cm}^{-1}$ is due to the O-H stretch, and the peak at 2938.04 $\mathrm{cm}^{-1}$ is assigned to the stretching vibrations of the $\mathrm{CH}_{2}$ functional group. The peaks at $1741.78 \mathrm{~cm}^{-1}$ and 1604.83 $\mathrm{cm}^{-1}$ are attributed to carbonyl stretching vibration, an initial methyl group, and amide I group of proteins, respectively. Finally, the peak at $1056.1 \mathrm{~cm}^{-1}$ is responsible for the stretching band of C-O groups [22]. The FTIR spectra of Ag-NPs synthesized from C. spinosa fruit extract is shown in Figure 2.B. The slight shifts in peak positions of the synthesized Ag-NPs from 3381.33,

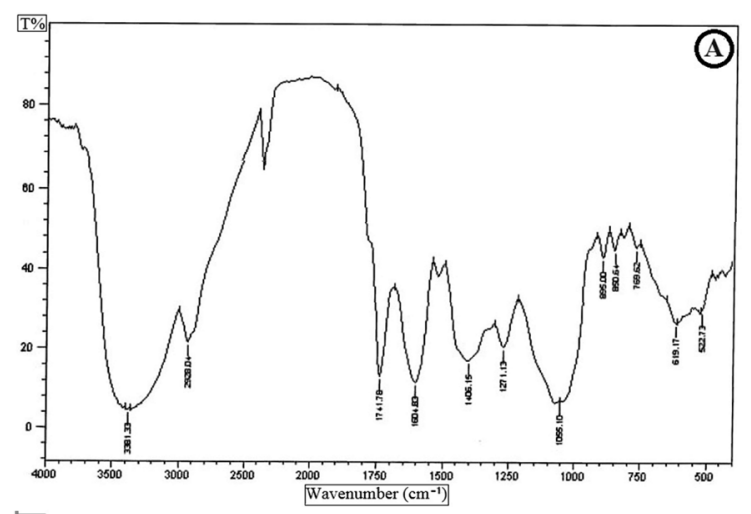

$2938.04,1741.78,1604.83$, and $1056.1 \mathrm{~cm}^{-1}$ to 3383.26 , 2936.04, 1612.54, 1518.04, and $1068.60 \mathrm{~cm}^{-1}$ respectively, indicates that different phytochemicals present in C. spinosa fruit extract are responsible for reducing and stabilizing the nanoparticles (Figure 2.B).

Scanning Electron Microscopy analysis of AgNPs

SEM images analyses can reveal the formation and morphology of stable Ag-NPs achieved from our green approach. Our scans showed the presence of both ag-

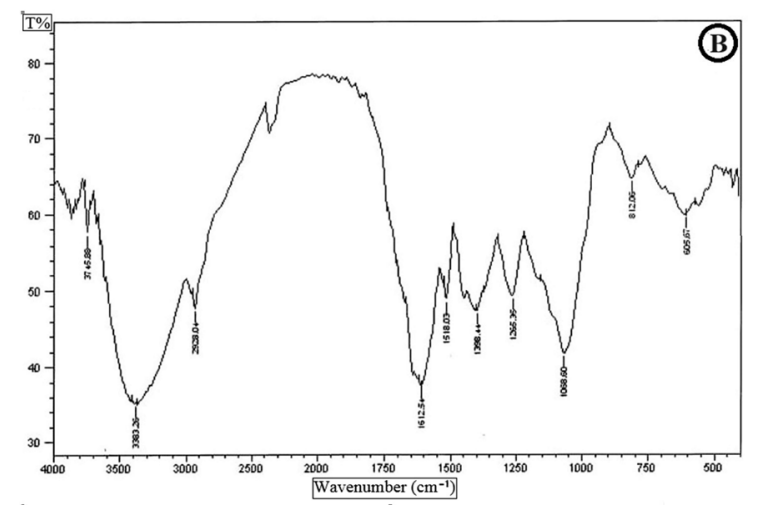

grmm

Figure 2. Fourier transforms infrared spectra of the A: water extract of C. spinosa fruit, and B: synthesized Ag-NPs from C. spinosa fruit extract 


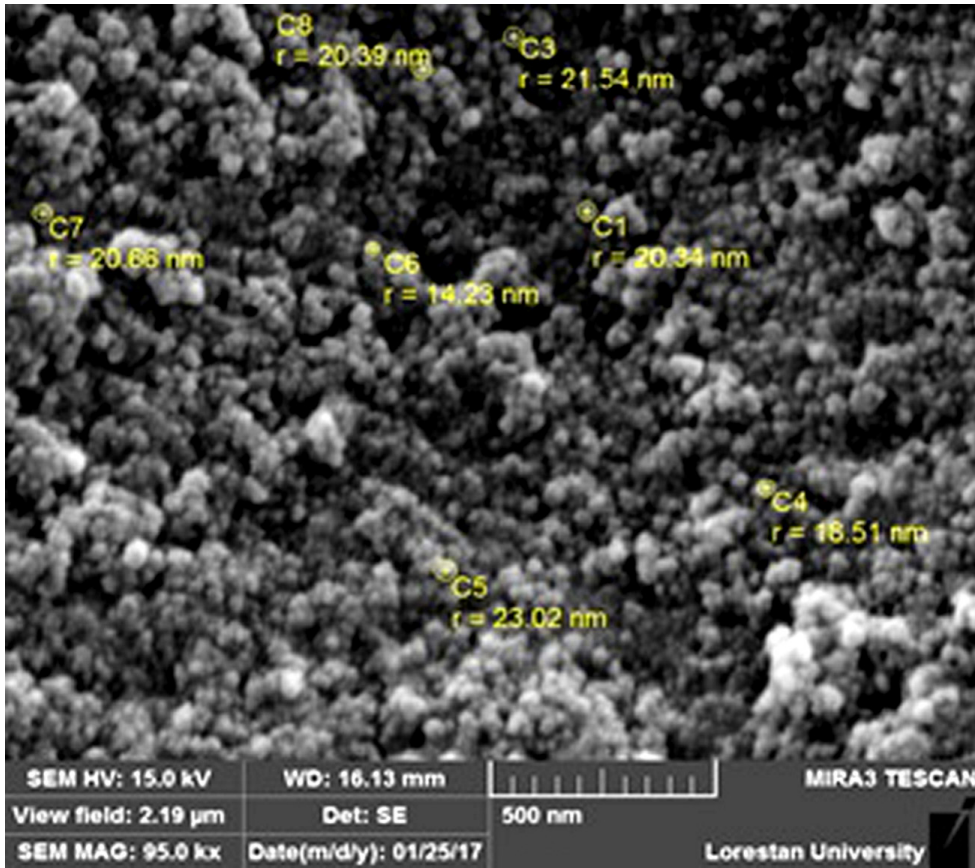

Figure 3. Scanning electron microscope micrograph of Ag-NPs synthesized by C. spinosa fruit extract

gregated and dispersed Ag-NPs. The nanoparticles were mainly uniform in a spherical shape with the average particle size from 50 to $80 \mathrm{~nm}$. The $\mathrm{K}_{\max }$ obtained from UV-Vis spectra for the distribution of size has an excellent resemblance with the SEM (Figure 3).

\section{Antifungal assays}

Silver ions, especially Ag-NPs, are known to have strong antimicrobial activities. Although our synthesized Ag-NPs showed weak antifungal activity compared with a standard antibiotic (fluconazole), it was effective to some extent against $\mathrm{C}$. albicans, $\mathrm{C}$. glabrata, and $K$. marxianus. It must be considered that the antifungal potential of Ag-NPs depends on the fungus type, the size of Ag-NPs, and associated closely with the formation of pits in the cell wall of fungus membrane integrity (Table 1).

\section{Discussion}

Since Ag-NPs have shown various pharmacological potential applications, including anti-cancer, anti-parasitic, antifungal, and antibacterial activities, the green synthesis of Ag-NPs offers additional advantages over other methods [9].

Comparing with physical and chemical methods, green biosynthesis of the NPs method is completely non-toxic and eco-friendly $[11,12]$. NPs have several antimicrobial mechanisms. For example, they affect respiratory cycle proteins and enzymes or generate free radicals and reactive oxygen in microbial cells [23]. In the case of Ag-NPs, they either anchor to the cell membrane, increase its permeability, and subsequently cause the cell death [24]; or create radicals and reactive oxygen, which may disrupt cellular integrity and normal functions. Artunduaga et al. [24] showed a potent antifungal activity of Ag-NPs against fluconazole-resistant Candida species. Artunduaga Bonilla et al. [24] synthesized efficient Ag-NPs using chemical reduction with cysteine with improved potency against $C$. krusei and C. glabrata compared with fluconazole.

In this study, we synthesized Ag-NPs from aqueous extract of $C$. spinosa fruit, which showed efficacy in reducing $\mathrm{Ag}^{2+}\left(\mathrm{AgNO}_{3}\right)$ bulk to $\mathrm{Ag}^{0}$ (Ag-NPs). Some potent compounds such as flavonoids, kaempferol, and anf thocyanins are present in aqueous extract of C. spinosa, which can act as a capping agent as well as an efficient stabilizer in the formation of NPs $[25,26]$.

Major parameters involved in biological function are size, monodispersity, and surface charge. Studies of Gguterrez-Martinez et al. showed that Ag-NPs could be the most potent antimicrobial agents when they are smaller than $100 \mathrm{~nm}$ in size [27]. Therefore, small NPs $(<100 \mathrm{~nm})$ have a variety of biological functions because of their increased surface area to volume ratio. Additionally, silver ions produced from Ag-NPs exhibit high reactivity to negatively charged functional groups in bio- 
logical molecules such as phospholipids, proteins, and polysaccharides [28].

In this study, the physical properties of synthesized NPs were extensively investigated using many techniques such as FTIR, SEM, and UV-Visible spectroscopy. Ag-NPs obtained from green synthesis were nearly spherical with the size ranging from 50 to $80 \mathrm{~nm}$. Our synthesized $\mathrm{Ag}$-NPs showed antifungal activity against $C$. albicans, C. glabra$t a$, and $K$. marxianus. Similarly, many studies have been conducted on the antifungal effects of Ag-NPs with different potencies. Nasrollahi et al. demonstrated that chemically synthesized $\mathrm{Ag}-\mathrm{NPs}$ by $\mathrm{PVP} / \mathrm{NaBH}_{4}$ had remarkable antifungal activity on C. albicans and S. cerevisiae [29].

Moazeni et al. [30] also showed that Ag-NPs synthesized by a fungal strain of the Trichophyton rubrum exhibited a strong impact against the growth of $C$. albicans. Kim et al. [31] reported that spherical Ag-NPs could effectively inhibit the growth of dermatophytes, including Trichophyton mentagrophites, Tricosporon begelli, and some Candida species even stronger than fluconazole.

\section{Conclusions}

Synthesis of Ag-NPs from aqueous extract of C. spino$s a$ fruit was done successfully, and a simple, quick, and inexpensive method for producing a desirable nanoparticle was designed. Our results showed that the produced Ag-NPs are in such suitable form, in size $(50-80 \mathrm{~nm})$ and spherical, that could be applied as antifungal agents for biological control of food spoiling and also as a new therapeutic agent against fungal infections in human and animals. Although compared with a standard antibiotic (fluconazole), the antifungal activity of synthesized AgNPs against $C$. albicans, $C$. glabrata, and $K$. marxianus was weak, it has low toxicity and cost-effective considering other biological advantages such as being biocompatible. This work was the first one to synthesis Ag-NPs from aqueous extract of $C$. spinos $a$ fruit. The synthesized Ag-NPs showed relatively acceptable antifungal activity against all fungi used in this study.

\section{Ethical Considerations}

\section{Compliance with ethical guidelines}

All ethical principles were considered in this article.

\section{Funding}

This study was funded by Payame Noor University, Khorramabad Branch, (Grant number: 994/27/1).

\section{Authors contribution's}

All authors contributed in preparing this article.

\section{Conflict of interest}

The authors declared no conflict of interest.

\section{Acknowledgements}

The authors would like to appreciate Payame Noor University, Khorramabad Branch, for sponsoring the project.

\section{References}

[1] Kabir MA, Hussain MA, Ahmad Z. Candida albicans: A model organism for studying fungal pathogens. ISRN Microbiol. 2012: 538694. [DOI:10.5402/2012/538694] [PMID] [PMCID]

[2] Mukherjee A, Pramanik S, Das D, Roy R, Therese KL. Polymicrobial chronic endophthalmitis diagnosed by culture and molecular technique. Indian J of Medic Microbiol. 2014; 32(3):331-2. [DOI:10.4103/0255-0857.136593] [PMID]

[3] Alka N, Priti S, Nilesh C, Hinal B, Binita D. Isolation of candida species in clinical specimens and its virulence factor: The biofilm. Int J of Medic Sci and Public Health. 2012; 1(2):97. [DOI:10.5455/ijmsph.2012.1.97-100]

[4] Romão D, Cavalheiro M, Mil-Homens D, Santos R, Pais P, Costa $\mathrm{C}$, et al. A new determinant of candida glabrata virulence: The acetate exporter cgdtr1. Front Cell Infect Microbiol. 2017; 7:473. [DOI:10.3389/fcimb.2017.00473] [PMID] [PMCID]

[5] Durán N, Silveira CP, Durán M, Martinez DS. Silver nanoparticle protein corona and toxicity: A mini-review. J Nanobiotechnol. 2015; 4(13):55. [DOI:10.1186/s12951-015-0114-4] [PMID] [PMCID]

[6] Furno F, Morley KS, Wong B, Sharp BL, Arnold PL, Howdle SM. Silver nanoparticles and polymeric medical devices: A new approach to prevention of infection. J Antimicrob Chemother. 2004, 54:1019-24. [DOI:10.1093/jac/dkh478] [PMID]

[7] Heydari R. Biological applications of biosynthesized silver nanoparticles through the utilization of plant extracts. Herb Med J. 2017; 2:87-95.

[8] Lai CW. Facile formation of colloidal silver nanoparticles using electrolysis technique and their antimicrobial activity. Micro Nano Lett. 2018; 13(3):407-10. [DOI:10.1049/mnl.2017.0805]

[9] Rai M, Ingle AP, Paralikar P, Gupta I, Medici S, Santos CA Recent advances in use of silver nanoparticles as antimalarial agents. Int J Pharm. 2017; 526(1-2):254-70. [DOI:10.1016/j.ijpp harm.2017.04.042] [PMID]

[10] Vazquez-Muñoz R, Borrego B, Juárez-Moreno K, GarcíaGarcía M, Mota MJD, Bogdanchikova N, et al. Toxicity of silver nanoparticles in biological systems: Does the complex- 
ity of biological systems matter. Toxicol Lett. 2017; 276:11-20. [DOI:10.1016/j.toxlet.2017.05.007] [PMID]

[11] Heydari R, Rashidipour M. Green synthesis of silver nanoparticles using extract of oak fruit hull (Jaft): Synthesis and in vitro cytotoxic effect on MCF-7 cells. Int J Breast Cancer. 2015; 846743. [DOI:10.1155/2015/846743] [PMID] [PMCID]

[12] Chandrakant KT, Sreekantha RD, Rohini A, Sungha P, Atul $\mathrm{K}$, Sushma S. Green synthesis of silver nanoparticles and their application for the development of optical fiber based hydrogen peroxide sensor. Sens. Actuator Biochem. 2013; 183:144-9. [DOI:10.1016/j.snb.2013.03.106]

[13] Zargari A. Medicinal Plants. Tehran: Tehran University Press; 1989.

[14] Ghahraman A. Chromophytes of Iran. Tehran: Tehran University Press; 1998.

[15] Mahmoudvand H, Ezatpour B, Jahanbakhsh S. The antileishmanial activity of essential oils from some traditionally used medicinal plants in iran. Herb Med J. 2016; 1(1):24-8.

[16] Biswas A, Vanlalveni C, Adhikari PP, Lalfakzuala R, Rokhum L. Green biosynthesis, characterisation and antimicrobial activities of silver nanoparticles using fruit extract of Solanum viarum. IET Nonobiotechnol. 2018; 12(7):933-8. [DOI:10.1049/ iet-nbt.2018.0050] [PMID]

[17] Sreemanti D, Jayeeta D, Asmita S, Soumya SB, Durba D, Anisur R. Khuda-Biosynthesized silver nanoparticles by ethanolic extracts of phytolacca decandra, gelsemium sempervirens, hydrastis canadesis and thuja occidentalis induce differential cytotaxicity through G2/M arrest in A375 cells. Colloids Surf B Biointerfaces. 2013; 101:325-36. [DOI:10.1016/j.cole surfb.2012.07.008] [PMID]

[18] Ebrahimi K, Shiravand S, Mahmoudvand H. Biosynthesis of copper nanoparticles using aqueous extract of Capparis spinosa fruit and investigation of its antibacterial activity. Marmara Pharm J. 2017; 21(4):866-71. [DOI:10.12991/mpj.2017.31]

[19] Sewell DL, Bove K, Callihan D. Protection of laboratory workers from occupationally acquired infections; Approved guideline-3rd ed (M29-A3). Wayne: Clinical and Laboratory Standards Institute; 2005.

[20] Falahati M, Sepahvand A, Mahmoudvand H, Baharvand P, Jabbarnia S, Ghojoghi A, et al. Evaluation of the antifungal activities of various extracts from Pistacia atlantica Desf. Curr Med Mycol. 2015; 1(3):25-32. [DOI:10.18869/acadpub. cmm.1.3.25] [PMID] [PMCID]

[21] Marzban A, Ebrahimipour G, Danesh A. Bioactivity of a novel glycolipid produced by a halophilic buttiauxella sp. and improving submerged fermentation using a response surface method. Molecules. 2016; 21:1256. [DOI:10.3390/molt ecules21101256] [PMID] [PMCID]

[22] Rodríguez-León E, Íñiguez-Palomares RA, Navarro RE, Rodríguez-Beas C, Larios-Rodríguez E, Alvarez-Cirerol FJ, et al. Silver nanoparticles synthesized with Rumex hymenosepalus extracts: Effective broad-spectrum microbicidal agents and cytotoxicity study. Artif Cells Nanomed Biotechnol. 2017; 46(6):1194-206. [DOI:10.1080/21691401.2017.1366332] [PMID]

[23] Henglein A. Physicochemical properties of small metal particles in solution: 'Microelectrode' reactions, chemisorption, composite metal particles, and the atom-to-metal transition. J Phys Chem. 1993; 97(21): 5457-71. [DOI:10.1021/j100123a004]
[24] Artunduaga Bonilla JJ, Paredes-Guerrero DJ, Sanchez-Suarez CI, Ortiz-Lopez CC, Torres-Saez RG. In vitro antifungal activity of silver nanoparticles against fluconazole candida species. World J Microbiol Biotechnol. 2015; 31(11):1801-9. [DOI:10.1007/s11274-015-1933-z] [PMID]

[25] Mansour RB, Jilani IB, Bouaziz M, Gargouri B, Elloumi N, Attia $\mathrm{H}$, et al. Phenolic contents and antioxidant activity of ethanolic extract of Capparis spinosa. Cytotechnology. 2016; 68(1):135-42 [DOI:10.1007/s10616-014-9764-6] [PMID] [PMCID]

[26] Gholami M, Shahzamani K, Marzban A, Lashgarian HE. Lashgarian H. E. Evaluation of antimicrobial activity of synthesised silver nanoparticles using Thymus kotschyanus aqueous extract. IET Nanobiotechnol. 2018; 12(8):1114-7 [DOI:10.1049/ietnbt.2018.5110] [PMID]

[27] Martinez-Gutierrez F, Olive PL, Banuelos A, Orrantia E, Nino $\mathrm{N}$, Sanchez EM, et al. Synthesis, characterization, and evaluation of antimicrobial and cytotoxic effect of silver and titanium nanoparticles. Nanomedicine. 2010; 6(5):681-8. [DOI:10.1016/j. nano.2010.02.001] [PMID]

[28] Kalwar K, Shan D. Antimicrobial effect of silver nanoparticles $(\mathrm{AgNPs})$ and their mechanism: A mini review. Micro Nano Lett. 2018; 13(3):277-80. [DOI:10.1049/mnl.2017.0648]

[29] Nasrollahi A, Pourshamsian KH, Mansourkiaee P. Antifungal activity of silver nanoparticles on some of fungi. Int J NanoDim. 2011; 1(3):233-9.

[30] Moazeni M, Rashidi N, Shahverdi AR, Noorbakhsh F, Rezaie S. Extracellular production of silver nanoparticles by using three common species of dermatophytes: Trichophyton rubrum, Trichophyton mentagrophytes and Microsporum canis. Iran Biomed J. 2012; 16(1):52-8. [PMID]

[31] Kim KJ, Sung WS, Moon SK, Choi JS, Kim JG, Lee DG. Antifungal effect of silver nanoparticles on dermatophytes. J Microbiol Biotechnol. 2008; 18(8):1482-4. [PMID] 
This Page Intentionally Left Blank 